\title{
SPECIAL LAGRANGIAN LINEAR SUBSPACES IN PRODUCT SYMPLECTIC SPACE
}

\author{
MAEGORZATA MIKOSZ \\ Faculty of Mathematics and Information Science, Warsaw University of Technology \\ Plac Politechniki 1, 00-661 Warszawa, Poland \\ E-mail: emmikosz@adam.mech.pw.edu.pl
}

\begin{abstract}
The notes consist of a study of special Lagrangian linear subspaces. We will give a condition for the graph of a linear symplectomorphism $f:\left(\mathbb{R}^{2 n}, \sigma=\sum_{i=1}^{n} d x_{i} \wedge d y_{i}\right) \rightarrow\left(\mathbb{R}^{2 n}, \sigma\right)$ to be a special Lagrangian linear subspace in $\left(\mathbb{R}^{2 n} \times \mathbb{R}^{2 n}, \omega=\pi_{2}^{*} \sigma-\pi_{1}^{*} \sigma\right)$. This way a special symplectic subset in the symplectic group is introduced. A stratification of special Lagrangian Grassmannian $S \Lambda_{2 n} \simeq S U(2 n) / S O(2 n)$ is defined.
\end{abstract}

1. Introduction. Symplectic manifold $(X, \alpha)$ is a $2 n$-dimensional manifold equipped with a closed differential form $\alpha$ such that $(\alpha)^{n}$ never vanish. A $k$-dimensional submanifold $Y \subset X$ is said to be isotropic if $\alpha$ restricted to every tangent plane $T_{x} Y, x \in Y$, vanish. In the case $k=n=\operatorname{dim} X / 2$ an isotropic submanifold is called Lagrangian. A diffeomorphism $f:(X, \alpha) \rightarrow(X, \alpha)$ is a symplectomorphism if $f^{*} \alpha=\alpha$. Recall that the graph of a symplectomorphism is a Lagrangian submanifold in the product $X \times X$ with the standard symplectic structure $\pi_{2}^{*} \alpha-\pi_{1}^{*} \alpha$, where $\pi_{1}, \pi_{2}$ are projections on arbitrary factors of $X \times X$. Kähler manifolds are distinguished class of symplectic manifolds. A manifold $(X, \alpha, \mathcal{J}, g)$ is said to be $K \ddot{K a h l e r}$ if $(X, \alpha)$ is a symplectic manifold, $\mathcal{J}$ a complex structure, $g$ a Hermitian metric on $X$ and $\alpha(u, \mathcal{J} v)$ is equal to the imaginary part of $g$. Let us assume that there exists a holomorphic $(n, 0)$-form $\Omega$ on $X$, in local coordinates $z_{1}, \ldots, z_{n} \in X$ the complex volume form and the symplectic form are equal to $\Omega=d z_{1} \wedge \ldots \wedge d z_{n}$ and $\alpha=\frac{i}{2} \sum_{k=1}^{n} d z_{k} \wedge d \bar{z}_{k}([\mathrm{MS}]$, [Wei]).

Definition 1.1. An oriented Lagrangian submanifold $L \subset(X, \alpha, \mathcal{J}, \Omega)$ is called special if $\left.\operatorname{Im} \Omega\right|_{L}=0$.

In fact there is a more general definition involving a phase $\theta \in[0,2 \pi]$. Let $\Lambda_{n}$ be the Lagrangian Grassmannian, i.e. a manifold consisting of all linear Lagrangian subspaces in $2 n$-dimensional linear symplectic space. Recall that $\Lambda_{n} \simeq U(n) / O(n)$, where $U(n)$ is the

2000 Mathematics Subject Classification: Primary 53D12, 51A50; Secondary 15A03, 53C38. Partially supported by RAAG and EAGER.

The paper is in final form and no version of it will be published elsewhere. 
unitary group and $O(n)$ the orthogonal group ([MS]). Let det : $\Lambda_{n} \rightarrow S^{1}$ be a mapping which sends a matrix $A$ representing a Lagrangian linear subspace $Y$ to its determinant, i.e. $\operatorname{det}(A)=\exp (i \theta)$. The number $\theta$ is called the phase of $Y$ ([HL], [Joy]). The mapping to $S^{1}$ can be defined globally if a global complex volume form $\Omega$ exists.

Definition 1.2. An oriented Lagrangian submanifold is said to be special if every its tangent space has the phase zero.

In a more general case special Lagrangian submanifolds with the fixed phase $\theta \in[0,2 \pi]$ are considered.

EXAMPLES.

1) In $\left(\mathbb{R}^{2}, \alpha=d x \wedge d y\right)$ the subspace $L=\{y=0\}$ is a linear special Lagrangian subspace with the phase 0 .

2) Recall that every Lagrangian submanifold can be locally described as the graph of a function differential. In $\mathbb{C}^{m}$ the condition for graph $d f\left(f: \mathbb{R}^{m} \rightarrow \mathbb{R}\right)$ to be a special Lagrangian submanifold is $\operatorname{Im} \operatorname{det}(I+i \operatorname{Hess} f)=0$, where $I$ is the identity matrix and Hess $f$ the Hessian of $f$. In general, the above condition is very difficult, this is a nonlinear second-order elliptic partial differential equation. For $m=2$ it gives the harmonic formula, i.e. $\Delta f=0$. For $m=3$ it has the form $\Delta f=\operatorname{det}(\operatorname{Hess} f)$ and this is the equation of Monge-Ampère type and its linearization at any solution is always elliptic ([HL]).

3) In $\mathbb{C}^{2}$ with the standard complex structure $\mathcal{I}: z_{0}=x_{0}+i x_{1}, z_{1}=x_{2}+i x_{3}$, every special Lagrangian submanifold is a $\mathcal{J}$-holomorphic curve with respect to the following structure $\mathcal{J}: w_{0}=x_{0}+i x_{2}, w_{1}=x_{1}-i x_{3} . \mathcal{J}$ is $\mathbb{R}$-linear and antiholomorphic, i.e. $\mathcal{J}(\mathcal{I} z)=-\mathcal{I}(\mathcal{J} z), z \in \mathbb{C}^{2}([$ Joy $])$.

The definition of a submanifold for which all tangent spaces have the common phase seems to be very restrictive. Special Lagrangian submanifolds can be defined only in symplectic manifolds $(X, \alpha, \mathcal{J})$ for which the holomorphic volume form is globally defined. Calabi-Yau manifolds, i.e. Kähler manifolds with the trivial canonical bundle (with global holomorphic volume form), have natural Lagrangian submanifolds which are special. Note that every special Lagrangian submanifold is a minimal submanifold, it minimizes the volume in its homology class. The special Lagrangian Grassmannian $S \Lambda_{n}$ (i.e. the family of all oriented $n$-dimensional vector subspaces in $2 n$-dimensional symplectic vector space $V$ ) can be identified with the quotient $S U(n) / S O(n)([\mathrm{HL}])$, where $S U(n)$ is the special unitary group and $S O(n)$ the special orthogonal group. If we consider the special linear Lagrangian subspace $L_{0}$ in $V$ spanned by the canonical basis $\left\{e_{1}, \ldots, e_{n}\right\}$ over real numbers, then every Lagrangian vector space in $V$ can be obtained by a unitary transformation of vectors $e_{1}, \ldots, e_{n}$ and every special Lagrangian vector space in $V$ can be produced by a special unitary transformation of $e_{1}, \ldots, e_{n}$. Special Lagrangian submanifolds are expected to play a role in the eventual explanation of Mirror Symmetry between Calabi-Yau manifolds (3-dimensional). Thus they are important in String Theory.

The paper is organized as follows. In the first part we give a condition for the graph of a linear symplectomorphism to be a special Lagrangian linear subspace. A special symplectic subset in the symplectic group is introduced. This subset consists of matrices representing linear symplectomorphisms whose graphs are special Lagrangian subspaces. We 
show some features of the special symplectic subset. In Section 3 we recall the stratification of the Lagrangian Grassmannian $\Lambda_{2 n} \simeq U(2 n) / O(2 n)$ constructed in the product of two symplectic spaces. The stratification is associated with a question when a Lagrangian subspace is or is not the graph of a linear symplectomorphism. It was defined by Janeczko ([Jan]). We introduce an analog of this partition in the special Lagrangian case. In the last part of the paper we show a partition of the special Lagrangian Grassmannian $S \Lambda_{2}$.

\section{Special Lagrangian subspaces as graphs of linear symplectomorphisms.}

We will consider the special Lagrangian geometry only in the linear case. Let $\left(\mathbb{R}^{2 n} \simeq\right.$ $\mathbb{C}^{n}, \sigma, \mathcal{J}, g$ ) be a linear symplectic space with a symplectic form $\sigma$, a Hermitian metric $g$, a complex structure $\mathcal{J}$, and $\sigma(u, \mathcal{J} v)$ be equal to the imaginary part of $g$. Let us endow the product $\left(\mathbb{C}^{n} \times \mathbb{C}^{n}, \omega,-\mathcal{J} \times \mathcal{J}\right)$ with the standard symplectic product structure $\omega=\pi_{2}^{*} \sigma-$ $\pi_{1}^{*} \sigma$, the complex structure $-\mathcal{J} \times \mathcal{J}$ compatible with $\omega$. We have the holomorphic volume form $\Omega=d \bar{z}_{1} \wedge \ldots \wedge d \bar{z}_{n} \wedge d z_{n+1} \wedge \ldots \wedge d z_{2 n}$ in local coordinates $\left(z_{1}, \ldots, z_{n}, z_{n+1}, \ldots, z_{2 n}\right)$.

We shall find a condition for a linear symplectomorphism to have the graph being a special Lagrangian linear subspace in $\left(\mathbb{C}^{2 n}, \omega\right)$.

LEMma 2.1. If $\mathcal{I} d:\left(\mathbb{C}^{n}, \sigma\right) \rightarrow\left(\mathbb{C}^{n}, \sigma\right)$ is the identity symplectomorphism, then

$$
\operatorname{phase}(\operatorname{graph} \mathcal{I} d)=n \frac{\pi}{2}(\bmod 2 \pi) \text {. }
$$

Proof. Let $\left(e_{1}, e_{2}, \ldots, e_{n},-i e_{1},-i e_{2}, \ldots,-i e_{n}\right)$ be the standard orthogonal basis of the domain $\left(\mathbb{C}^{n} \simeq \mathbb{R}^{2 n}, \sigma, \mathcal{J}\right)$ over $\mathbb{R}$, then $\left(e_{1}, \ldots, e_{n}, i e_{1}, \ldots, i e_{n}\right)$ is the image of the basis. Thus $L=\operatorname{graph} \mathcal{I} d$ is a real linear subspace spanned (over $\mathbb{R}$ ) by the columns of the matrix $\left(\begin{array}{cc}I & -i I \\ I & i I\end{array}\right)$, where the block $I$ is the identity matrix of dimension $n \times n$. We calculate: phase $(\operatorname{graph} \mathcal{I} d)=\arg (\operatorname{det} \operatorname{graph} \mathcal{I} d)=\arg \left((2 i)^{n}\right)=n \frac{\pi}{2}(\bmod 2 \pi)$.

The above result permits us to fix the phase $\theta=n \frac{\pi}{2}(\bmod 2 \pi)$ for special Lagrangian subspaces and submanifolds.

Proposition 2.2. Let $\Phi:\left(\mathbb{C}^{n}, \sigma\right) \rightarrow\left(\mathbb{C}^{n}, \sigma\right)$ be a linear real symplectomorphism and let the symplectic matrix $\Phi \in \operatorname{Sp}(n)$ have the block form $\Phi=\left(\begin{array}{ll}A & B \\ C & D\end{array}\right)$, where every block is a submatrix of dimension $n \times n$. Then graph $\Phi$ is a special Lagrangian linear subspace in $\left(\mathbb{C}^{n} \times \mathbb{C}^{n}, \omega\right)$ if and only if

$$
\arg \operatorname{det}((A+D)+i(C-B))=0
$$

Proof. We consider the orthogonal basis $\left(e_{1}, \ldots, e_{n},-i e_{1}, \ldots,-i e_{n}\right)$ as in the above lemma, then the matrix representing graph $\Phi$ as a real linear subspace in $\mathbb{C}^{n} \times \mathbb{C}^{n}$ is

$$
\operatorname{graph} \Phi=\left(\begin{array}{cc}
I & -i I \\
A+i C & B+i D
\end{array}\right)
$$

We calculate $\operatorname{det}(\operatorname{graph} \Phi)=\operatorname{det}((B-C)+i(A+D))=\operatorname{det}(i I) \operatorname{det}((A+D)+$ $i(C-B))=(i)^{n} \operatorname{det}((A+D)+i(C-B))$, thus $\arg (\operatorname{det}(\operatorname{graph} \Phi))=n \frac{\pi}{2}(\bmod 2 \pi)$ if and only if $\operatorname{det}((A+D)+i(C-B)) \in \mathbb{R}_{+}$.

A special Lagrangian subspace should have fixed orientation. If we consider graph $\Phi$ with the opposite orientation we deduce that graph $\Phi$ is a special Lagrangian subspace if and only if $\operatorname{det}((A+D)+i(C-B)) \in \mathbb{R}_{-}$. 
REMARK 2.3. The graph of a linear symplectomorphism is a special Lagrangian linear subspace if after choosing an arbitrary orientation the determinant of the matrix $((A+D)+i(C-B))$ is a real number.

We can express the conditions in terms of complex structure $\mathcal{J}$. Define

$$
\Phi+\mathcal{J} \Phi \mathcal{J}^{-1}=\Phi+\left(\Phi^{T}\right)^{-1}=\left(\begin{array}{ll}
A+D & B-C \\
C-B & A+D
\end{array}\right) \simeq((A+D)+i(C-B)),
$$

where $\Phi=\left(\begin{array}{ll}A & B \\ C & D\end{array}\right) \in \operatorname{Sp}(n)$. We have used the identification between matrices over $\mathbb{R}$ and over $\mathbb{C}$, i.e. $(X+i Y) \simeq\left(\begin{array}{cc}X & -Y \\ Y & X\end{array}\right)$, $\operatorname{det}(X+i Y)=\left|\operatorname{det}\left(\begin{array}{cc}X & -Y \\ Y & X\end{array}\right)\right|^{2}$.

DeFinition 2.4. A special symplectic subset is the subset in the symplectic group $\operatorname{Sp}(n)$ consisting of the matrices whose graph is a special Lagrangian subspace, we denote it by $\operatorname{SSp}(n)$.

Obviously $\operatorname{SSp}(n)$ is not a subgroup in $\operatorname{Sp}(n)$.

EXAMPLE 2.5. We consider the symplectic group in $\mathbb{C} \simeq \mathbb{R}^{2}$, i.e. $\operatorname{Sp}(1) \simeq \operatorname{SL}(2, \mathbb{R})$ $(\mathrm{SL}(2, \mathbb{R})$ - special linear group). The special symplectic subset in $\operatorname{Sp}(1)$ consists of symmetric and positive definite matrices: $\operatorname{SSp}(1)=\left\{\Phi=\left(\begin{array}{ll}a & b \\ b & d\end{array}\right): a+d>0, \Phi \in \operatorname{Sp}(1)\right\}$. We see that $\operatorname{SSp}(1)$ is not a subgroup in $\operatorname{Sp}(1)$.

EXAMPLE 2.6. We will show that the matrix $\Phi=\left(\begin{array}{ll}2 & 1 \\ 1 & 1\end{array}\right) \in \operatorname{SSp}(1)$ represents the linear symplectomorphism $f:(\mathbb{C}, \sigma) \rightarrow(\mathbb{C}, \sigma)$ whose graph is the special Lagrangian vector subspace in $\left(\mathbb{C} \times \mathbb{C}, \omega=\pi_{2}^{*} \sigma-\pi_{1}^{*} \sigma,-i \times i, \Omega=d \bar{z}_{1} \wedge d z_{2}\right)$, i.e. the phase of graph $f$ is $\frac{\pi}{2}$.

Let $e_{1}=(1,0) \simeq 1, e_{2}=(0,1) \simeq i$ be the canonical basis of $\mathbb{C} \simeq \mathbb{R}^{2}$. We calculate that $f\left(e_{1}\right)=\Phi e_{1}=(2,1) \simeq 2+i$ and $f\left(e_{2}\right)=\Phi e_{2}=(1,1) \simeq 1+i$. Thus graph $\Phi$ is a Lagrangian vector subspace in $\mathbb{C} \times \mathbb{C}$ represented by the matrix

$$
\operatorname{graph} \Phi=\left(\begin{array}{ll}
1 & 0 \\
0 & 1 \\
2 & 1 \\
1 & 1
\end{array}\right) \simeq\left(\begin{array}{cc}
1 & -i \\
2+i & 1+i
\end{array}\right)
$$

We calculate that $\omega([1,0,2,1],[0,1,1,1])=\sigma([2,1],[1,1])-\sigma([1,0],[0,1])=-1+1=0$ and $\Omega([1,0,2,1],[0,1,1,1])=\operatorname{det}\left(\begin{array}{cc}1 & -i \\ 2+i & 1+i\end{array}\right)=3 i$. Thus phase $(\operatorname{graph} f)=\frac{\pi}{2}$.

We will show some properties of $\operatorname{SSp}(n)$.

\section{REMARK 2.7.}

1) Every special symplectic matrix $\Phi \in \operatorname{SSp}(n)$ can be decomposed as $\Phi=P Q$, where $P$ is symmetric, symplectic and positive definite, and $Q \in S U(n)$. The subgroup $S U(n)$ is a maximal compact subset in $\operatorname{SSp}(n)$, like $U(n)$ in $\operatorname{Sp}(n)$.

2) $\operatorname{phase}(\operatorname{graph} \Phi)-n \frac{\pi}{2}=-\left(\operatorname{phase}\left(\operatorname{graph} \Phi^{-1}\right)-n \frac{\pi}{2}\right)$.

3) $\operatorname{phase}\left(\operatorname{graph} \Phi^{T}\right)=\operatorname{phase}\left(\operatorname{graph} \Phi^{-1}\right)$. 
4) $\Phi S U(n) \subset \operatorname{SSp}(n)$ and $S U(n) \Phi \subset \operatorname{SSp}(n)$ for $\Phi \in \operatorname{SSp}(n)(\Phi S U(n)$ and $S U(n) \Phi$ can be treated as „cosets” of $S U(n)$ in $\operatorname{SSp}(n))$.

Proof. We know that $\Phi \in \operatorname{SSp}(n)$ like every symplectic matrix is a product of two matrices, where the first is symmetric, symplectic and positive definite and the second is a unitary matrix $([\mathrm{MS}])$; $\operatorname{det}\left(\Phi+\mathcal{J} \Phi \mathcal{J}^{-1}\right)=\operatorname{det}\left(P+\mathcal{J} P \mathcal{J}^{-1}\right) \operatorname{det} Q$. Analyzing eigenvalues of $P$ and $\mathcal{J} P \mathcal{J}^{-1}$, we see that $\operatorname{det}\left(P+\mathcal{J} P \mathcal{J}^{-1}\right) \in \mathbb{R}_{+}$thus $\operatorname{det} Q=1$.

3. Stratification of the special Lagrangian Grassmannian. We will explore the Lagrangian Grassmannian in the Cartesian product of two copies of a linear symplectic space. We recall very natural stratification of $\Lambda_{2 n}$ introduced in [Jan]. The partition is associated to a question when a Lagrangian subspace is or is not the graph of a linear symplectomorphism. Next we introduce an analogous stratification in the special Lagrangian Grassmannian $S \Lambda_{2 n}$.

A very easy and useful observation leads us to the stratification of $\Lambda_{2 n}$ ([Jan]).

REMARK 3.1. If $L \subset\left(\mathbb{C}^{n} \times \mathbb{C}^{n}, \omega=\pi_{2}^{*} \sigma-\pi_{1}^{*} \sigma,-\mathcal{J} \times \mathcal{J}\right)$ is a linear Lagrangian subspace, then there are two possibilities:

1) $L$ is transversal to $\mathbb{C}^{n} \times\{0\}$ and to $\{0\} \times \mathbb{C}^{n}$ simultaneously or

2) $L$ is transversal neither to $\mathbb{C}^{n} \times\{0\}$ nor to $\{0\} \times \mathbb{C}^{n}$ and always $\operatorname{codim} \pi_{1}(L)=\operatorname{codim} \pi_{2}(L)$.

This condition divides Grassmannian $\Lambda_{2 n}$ into two parts: the regular part consisting of the graphs of linear symplectomorphisms and the critical stratum which contains the graphs of linear symplectic correspondences.

In fact the stratification is $\Lambda_{2 n}=R \Lambda_{2 n}+\sum_{k=1}^{n} C_{k} \Lambda_{2 n}$, where

- $R \Lambda_{2 n}$ is the regular stratum, if $L \in R \Lambda_{2 n}$ then $\operatorname{codim} \pi_{1}(L)=\operatorname{codim} \pi_{2}(L)=0$,

- $\sum_{k=1}^{n} C_{k} \Lambda_{2 n}$ is the critical set; if $L \in C_{k} \Lambda_{2 n}$ then $\operatorname{codim} \pi_{1}(L)=\operatorname{codim} \pi_{2}(L)=k$.

Proposition 3.2. In the case of special Lagrangian Grassmannian we have an analogous partition, only the deepest stratum is different:

$$
S \Lambda_{2 n}=R S \Lambda_{2 n}+\sum_{k=1}^{n-1} C_{k} S \Lambda_{2 n}+C_{n} S \Lambda_{2 n} .
$$

1) The regular stratum $R S \Lambda_{2 n}$ consists of the graphs of special linear symplectomorphisms $\mathbb{C}^{n} \rightarrow \mathbb{C}^{n}$, therefore it can be identified with two copies of the special symplectic subset (two orientations are possible)

$$
R S \Lambda_{2 n} \simeq \operatorname{SSp}^{+}(n) \sqcup \mathrm{SSp}^{-}(n) .
$$

2) Every stratum $C_{k} S \Lambda_{2 n}, k=1, \ldots, n-1$, is fibered in the following way:

$$
\begin{aligned}
\operatorname{SSp}^{+}(n-k) \sqcup \operatorname{SSp}^{-}(n-k) \hookrightarrow & C_{k} S \Lambda_{2 n} \\
& \downarrow \\
& \mathcal{I}_{k}^{2 n} \times \mathcal{I}_{k}^{2 n}
\end{aligned}
$$

where $\mathcal{I}_{k}^{2 n}$ denotes the isotropic Grassmannian, i.e. the set of all $k$-dimensional isotropic linear subspaces in $\mathbb{C}^{n} \simeq \mathbb{R}^{2 n}$. Recall that $\mathcal{I}_{k}^{2 n} \simeq U(n) /(O(k) \oplus U(n-k))$, where $U(n)$ 
and $U(n-k)$ are the unitary groups and $O(k)$ the orthogonal group ([MS]). In the above bundle we have the projection on symplectic polars and fibers consist of linear symplectomorphisms between reduced symplectic spaces $\pi_{1}(L) / \pi_{1}(L)^{\perp} \rightarrow \pi_{2}(L) / \pi_{2}(L)^{\perp}$.

3) Supercritical stratum $C_{n} S \Lambda_{2 n}$ is a subset of $\Lambda_{n} \times \Lambda_{n}$. If $L \in C_{n} S \Lambda_{2 n}$ then $L=$ $L_{1} \times L_{2}, L_{i} \in \Lambda_{n}, i=1,2$, and $\operatorname{phase}\left(L_{1} \times L_{2}\right)=n \frac{\pi}{2}(\bmod 2 \pi)$ or $\operatorname{phase}\left(L_{1} \times L_{2}\right)=$ $n \frac{\pi}{2}+\pi(\bmod 2 \pi)$ if an orientation of the subspace $L_{1}$ or $L_{2}$ is changed.

Proof. Items 1), 2) are obvious ([Jan]). In 3) phase $\left(L_{1} \times L_{2}\right)=\arg \left(\overline{\operatorname{det} L_{1}} \operatorname{det} L_{2}\right)=$ $\arg \left(\exp \left(-i \theta_{1}\right) \exp i \theta_{2}\right)=$ phase $L_{2}-$ phase $L_{1}=\theta_{2}-\theta_{1}=n \frac{\pi}{2}(\bmod 2 \pi)$.

4. Example. We will explore the stratification of the special Lagrangian Grassmannian $S \Lambda_{2 n}$ in the smallest interesting dimension, $2 n=2$. Recall that $S \Lambda_{2}$ is the family of special real Lagrangian subspaces in $\left(\mathbb{C}^{2}, \mathcal{J}, \omega\right), \operatorname{dim} S \Lambda_{2}=2$ and $S \Lambda_{2} \simeq S U(2) / S O(2) \simeq$ $S^{3} / S^{1} \simeq S^{2}$.

The Grassmannian $S \Lambda_{2}$ can be divided into two strata:

1) the regular stratum $R S \Lambda_{2}$ which consists of the graphs of linear symplectomorphisms $\Phi: \mathbb{C} \rightarrow \mathbb{C}$,

2) the critical stratum $C_{1} S \Lambda_{2}$ which is included in the Cartesian product $\Lambda_{1} \times \Lambda_{1}$, where $\Lambda_{1} \simeq U(1) / O(1) \simeq S^{1}$ denotes the Lagrangian Grassmannian in $\mathbb{R}^{2} \simeq \mathbb{C}$.

Using our results from Proposition 3.2 we see that the open stratum consists of two copies of special symplectic subset $\operatorname{SSp}(1)$ (see Example 2.5), i.e. $R S \Lambda_{2} \simeq \mathrm{SSp}^{+}(1) \sqcup$ $\operatorname{SSp}^{-}(1)$. If $L \in C_{1} S \Lambda_{2}$ then $L=L_{1} \times L_{2}$, phase $\left(L_{1} \times L_{2}\right)=\pi / 2$, thus the matrix $\Phi \in U(1) \times U(1)$ representing $L$ is

$$
\Phi=\left(\begin{array}{cc}
\exp \left(-i \theta_{1}\right) & 0 \\
0 & \exp \left(i \theta_{2}\right)
\end{array}\right), \quad \theta_{2}-\theta_{1}=\frac{\pi}{2} .
$$

The stratum $C_{1} S \Lambda_{2}$ can be identified with the circle $S^{1}$ on the torus $\Lambda_{1} \times \Lambda_{1} \simeq$ $S^{1} \times S^{1} \simeq T^{2}$. How are the strata located on the sphere $S^{2} \simeq S \Lambda_{2}$ ? The stratum $C_{1} S \Lambda_{2} \simeq S^{1}$ is the equator and $R S \Lambda_{2}$ two hemispheres. If we use coordinates $s, \delta, b$ which describe the set $\mathrm{SSp}(1)$ in Example $2.5\left(s=\frac{a+d}{2}, a=s-\delta, d=s+\delta\right)$ and we parametrize $\operatorname{SSp}(1)$ by $t$ and $\gamma: s=\cosh t, \delta=\sinh t \cos \gamma, b=\sinh t \sin \gamma$, we can show that if $t \rightarrow \infty$ then graph $\Phi(t, \gamma) \rightarrow\left(-\gamma, \gamma+\frac{\pi}{2}\right)$. It means that the deepest stratum is attached to a bigger one.

\section{References}

[HL] R. Harvey, H. B. Lawson Jr., Calibrated geometries, Acta Math. 148 (1982), 47-157.

[Jan] S. Janeczko, Lagrangian submanifolds in product symplectic space, J. Math. Phys. 41 (2000), 5642-5655.

[Joy] D. D. Joyce, Lectures on special Lagrangian geometry, Proceedings of the Clay Mathematics Institute Summer School on Global Theory of Minimal Surfaces, MSRI, Berkeley, 2001, (arXiv:math.DG/0111111).

[MS] D. McDuff, D. Salamon, Introduction to Symplectic Topology, The Clarendon Press, Oxford Univ. Press, New York, 1995.

[Wei] A. Weinstein, Lectures on Symplectic Manifolds, CBMS Regional Conf. Ser. in Math. 29, Amer. Math. Soc., Providence, 1977. 